Research Paper

\title{
ADVANCIS Score Predicts Acute Kidney Injury After Percutaneous Coronary Intervention for Acute Coronary Syndrome
}

\author{
Pei-Chun Fan ${ }^{1,2,5^{*}}$, Tien-Hsing Chen ${ }^{2,3^{*}}$, Cheng-Chia Lee ${ }^{1,2,5}$, Tsung-Yu Tsai ${ }^{1,2,5}$, Yung-Chang Chen ${ }^{2,4}$, \\ Chih-Hsiang Chang $1,2,5 \bowtie$
}

1. Department of Nephrology, Kidney Research Center, Chang Gung Memorial Hospital, Linkou Medical Center, Chang Gung University, No. 5 Fusing Street, Gueishan Dist., Taoyuan City 333, Taiwan (R.O.C.)

2. College of Medicine, Chang Gung University, No.259, Wenhua 1st Rd., Guishan Dist., Taoyuan City 33302, Taiwan (R.O.C.)

3. Department of Cardiology, Chang Gung Memorial Hospital, Keelung Branch, No. 222, Maijin Rd., Anle Dist., Keelung City 20401, Taiwan (R.O.C.)

4. Department of Nephrology, Chang Gung Memorial Hospital, Keelung Branch, No. 222, Maijin Rd., Anle Dist., Keelung City 20401, Taiwan (R.O.C.)

5. Graduate Institute of Clinical Medical Sciences, Chang Gung University, No.259, Wenhua 1st Rd., Guishan Dist., Taoyuan City 33302, Taiwan (R.O.C.)

* Pei-Chun Fan and Tien-Hsing Chen contributed equally to this manuscript

$\square$ Corresponding author: Chih-Hsiang Chang, MD., Kidney research center, Nephrology department, Chang-Gung Memorial Hospital, Chang-Gung University College of Medicine, No. 5 Fusing Street, Gueishan Dist., Taoyuan City 333, Taiwan (R.O.C.) E-mail: franwisandsun@gmail.com; Phone No.: 886-3-3281200 ext 8181; Fax: $886-3-3282173$

(c) Ivyspring International Publisher. This is an open access article distributed under the terms of the Creative Commons Attribution (CC BY-NC) license (https:// creativecommons.org/licenses/by-nc/4.0/). See http://ivyspring.com/terms for full terms and conditions.

Received: 2017.09.28; Accepted: 2018.02.02; Published: 2018.03.08

\begin{abstract}
Acute kidney injury (AKI), a common and crucial complication of acute coronary syndrome (ACS) after receiving percutaneous coronary intervention (PCl), is associated with increased mortality and adverse outcomes. This study aimed to develop and validate a risk prediction model for incident $\mathrm{AKI}$ after $\mathrm{PCl}$ for ACS. We included 82,186 patients admitted for ACS and receiving $\mathrm{PCl}$ between 1997 and 2011 from the Taiwan National Health Insurance Research Database and randomly divided them into a training cohort $(n=57,630)$ and validation cohort $(n=24,656)$ for risk model development and validation, respectively. Risk factor analysis revealed that age, diabetes mellitus, ventilator use, prior AKI, number of intervened vessels, chronic kidney disease (CKD), intra-aortic balloon pump (IABP) use, cardiogenic shock, female sex, prior stroke, peripheral arterial disease, hypertension, and heart failure were significant risk factors for incident $\mathrm{AKI}$ after $\mathrm{PCI}$ for ACS. The reduced model, ADVANCIS, comprised 8 clinical parameters (age, diabetes mellitus, ventilator use, prior AKI, number of intervened vessels, CKD, IABP use, cardiogenic shock), with a score scale ranging from 0 to 22, and performed comparably with the full model (area under the receiver operating characteristic curve, $87.4 \%$ vs $87.9 \%$ ). An ADVANCIS score of $\geq 6$ was associated with higher in-hospital mortality risk. In conclusion, the ADVANCIS score is a novel, simple, robust tool for predicting the risk of incident $\mathrm{AKI}$ after $\mathrm{PCl}$ for $\mathrm{ACS}$, and it can aid in risk stratification to monitor patient care.
\end{abstract}

Key words: Acute kidney injury; Acute coronary syndrome; Percutaneous coronary intervention; Mortality; Prediction model

\section{Introduction}

Acute kidney injury (AKI) is a common complication of critical illnesses, including acute coronary syndrome (ACS). The reported incidence of AKI is approximately $12.0 \%-36.6 \%$ [1-5]. In patients with ACS, AKI is associated with significantly increased mortality and morbidity $[1-3,6]$. Notably, the severity and duration of AKI are correlated with the risk of chronic kidney disease (CKD) $[7,8]$. 
Many factors contribute to the development of AKI following ACS [9, 10], including altered haemodynamics secondary to impaired cardiac output, contrast media exposure, neurohormonal activation, inflammation, oxidative stress, bleeding, acidosis, and hyperglycaemia. In addition, percutaneous coronary intervention (PCI) and intra-aortic balloon pump (IABP) use predispose patients to atheroembolism. Many medications, such as diuretics, angiotensin-converting-enzyme inhibitors (ACEis), angiotensin II receptor blockers (ARBs), nonsteroidal anti-inflammatory drugs, antibiotics, and vasopressors, may aggravate kidney injury.

Despite advances in research over the past decades, effective treatments for AKI are not available. Prevention and early intervention remain the most effective strategies for AKI of any entity. To date, many individual risk factors for AKI after ACS have been reported [2, 4, 11]. However, the cumulative risk, obtained by combining multiple risk factors, has not been assessed. A practical clinical tool to predict the risk of post-ACS AKI is not available. This study developed a risk-prediction model for incident AKI after PCI for ACS, for enabling clinicians to identify high-risk patients, thereby facilitating effective prevention, prompt intervention for severity reduction, and improvement of clinical outcomes.

\section{Results}

\section{Patient characteristics}

Table 1 and Table 2 summarize the basic characteristics and clinical information of the training and validation cohorts. The training cohort comprised 57,530 patients, among which 44,785 (77.8\%) were male, the mean age was 63.9 years, and 2,670 (4.6\%) patients had incident AKI. The validation cohort comprised 24,656 patients, among which 19,077 $(77.4 \%)$ were male, the mean age was 64.0 years, and 1,159 (4.7\%) patients had incident AKI.

Overall, 19331 patients $(23.5 \%)$ experienced cardiogenic shock and 9,417 patients $(11.5 \%)$ received IABP installation. The in-hospital mortality rate was $6.5 \%(33.8 \%$ in patients with AKI, $5.1 \%$ in patients without AKI). The rate of de novo dialysis requirement in the AKI patients was $60.5 \%$ and $56.6 \%$ in the training and validation cohorts, respectively.

In both cohorts, the patients with AKI were more likely to be female, be older, and have a higher prevalence of comorbidities, such as diabetes mellitus, hypertension, coronary artery disease, prior myocardial infarction, heart failure, CKD, prior AKI, prior stroke, peripheral arterial disease, gout, and malignancy.
The patients with AKI had a higher number of intervened vessels and were more likely to experience cardiogenic shock, receive IABP installation, receive ventilator support, receive a higher inotropic dosage, and receive blood transfusion secondary to major bleeding. The patients with AKI had a longer hospital stay and intensive care unit (ICU) stay than did the patients without AKI.

Table 1. Baseline characteristics and clinical information in the training and validation cohorts

\begin{tabular}{|c|c|c|c|}
\hline Characteristics & $\begin{array}{l}\text { Training } \\
(N=57,530)\end{array}$ & $\begin{array}{l}\text { Validation } \\
(N=24,656)\end{array}$ & $p$ \\
\hline Age (years) & & & 0.064 \\
\hline$<75$ years & $43,994(76.5)$ & $18,707(75.9)$ & \\
\hline$\geq 75$ years & $13,536(23.5)$ & $5,949(24.1)$ & \\
\hline Male & 44,785 (77.8) & $19,077(77.4)$ & 0.135 \\
\hline \multicolumn{4}{|l|}{ Comorbidities } \\
\hline Diabetes mellitus & $20,089(34.9)$ & $8,567(34.7)$ & 0.633 \\
\hline Hypertension & $30,847(53.6)$ & $13,297(53.9)$ & 0.412 \\
\hline Coronary artery disease & $8,385(14.6)$ & $3,648(14.8)$ & 0.412 \\
\hline Prior myocardial infarction & $6,615(11.5)$ & 2,768 (11.2) & 0.261 \\
\hline Heart failure & $3,804(6.6)$ & $1,677(6.8)$ & 0.319 \\
\hline Chronic kidney disease & $1,255(2.2)$ & $555(2.3)$ & 0.534 \\
\hline Prior AKI & $870(1.5)$ & $358(1.5)$ & 0.514 \\
\hline Prior stroke & $5,989(10.4)$ & $2,536(10.3)$ & 0.591 \\
\hline Peripheral arterial disease & $2,023(3.5)$ & $950(3.9)$ & 0.018 \\
\hline Gout & $4,343(7.5)$ & $1,823(7.4)$ & 0.438 \\
\hline Malignancy & $2,421(4.2)$ & $1,005(4.1)$ & 0.385 \\
\hline Number of intervened vessels & & & 0.995 \\
\hline 1 & $46,892(81.5)$ & 20,097 (81.5) & \\
\hline 2 & $9,496(16.5)$ & $4,067(16.5)$ & \\
\hline 3 & $1,142(2.0)$ & $492(2.0)$ & \\
\hline Cardiogenic shock & $13,593(23.6)$ & $5,738(23.3)$ & 0.271 \\
\hline IABP use & 6,684 (11.6) & 2,733 (11.1) & 0.028 \\
\hline Ventilator use & $6,228(10.8)$ & 2,597 (10.5) & 0.214 \\
\hline \multicolumn{4}{|l|}{ Dosage of inotropic medications } \\
\hline Dopamine $\left(\times 10^{3} \mathrm{mg}\right)$ & $0.5 \pm 2.4$ & $0.5 \pm 2.2$ & 0.633 \\
\hline Norepinephrine (mg) & $0.6 \pm 4.7$ & $0.6 \pm 3.8$ & 0.080 \\
\hline Epinephrine (mg) & $2.6 \pm 23.0$ & $2.8 \pm 39.1$ & 0.496 \\
\hline \multicolumn{4}{|l|}{ Other medications } \\
\hline Aspirin & $54,310(94.4)$ & $23,234(94.2)$ & 0.333 \\
\hline Clopidogrel & $51,372(89.3)$ & $22,192(90.0)$ & 0.002 \\
\hline B-blocker & $36,287(63.1)$ & $15,721(63.8)$ & 0.061 \\
\hline ACEi/ARB & $43,315(75.3)$ & $18,695(75.8)$ & 0.104 \\
\hline Calcium channel blocker & $17,783(30.9)$ & $7,517(30.5)$ & 0.228 \\
\hline Statin & $27,133(47.2)$ & $11,808(47.9)$ & 0.056 \\
\hline PPI & 4,333 (7.5) & $1,883(7.6)$ & 0.601 \\
\hline GP IIb/IIIa & $1,082(1.9)$ & $452(1.8)$ & 0.645 \\
\hline Metformin & $8,451(14.7)$ & 3,620 (14.7) & 0.977 \\
\hline ICU stays (days) & $4.2 \pm 6.7$ & $4.2 \pm 7.3$ & 0.457 \\
\hline Hospital stays (days) & $9.3 \pm 16.5$ & $9.3 \pm 14.9$ & 0.684 \\
\hline In hospital mortality & $3,746(6.5)$ & $1,572(6.4)$ & 0.469 \\
\hline $\begin{array}{l}\text { Major bleeding requiring blood } \\
\text { transfusion }\end{array}$ & $9,949(17.3)$ & $4,242(17.2)$ & 0.757 \\
\hline
\end{tabular}

$\mathrm{ACEi}$, angiotensin converting enzyme inhibitor; $\mathrm{AKI}$, acute kidney injury; ACS, acute coronary syndrome; ARB, angiotensin II receptor blocker; GP, glycoprotein; IABP, intra-aortic balloon pump; ICU, intensive care unit; PPI, proton pump inhibitor. 
Table 2. Baseline characteristics and clinical information in the training and validation cohorts with and without AKI

\begin{tabular}{|c|c|c|c|c|c|c|}
\hline \multirow[b]{2}{*}{ Characteristics } & \multicolumn{3}{|c|}{ Training cohort $(N=57,530)$} & \multicolumn{3}{|c|}{ Validation cohort $(N=24,656)$} \\
\hline & $\begin{array}{l}\text { AKI } \\
(N=2,670)\end{array}$ & $\begin{array}{l}\text { Non-AKI } \\
(N=54,860)\end{array}$ & $p$ & $\begin{array}{l}\text { AKI } \\
(N=1,159)\end{array}$ & $\begin{array}{l}\text { Non-AKI } \\
(N=23,497)\end{array}$ & $p$ \\
\hline Age (years) & $71.1 \pm 11.7$ & $63.5 \pm 13.2$ & $<0.001$ & $70.8 \pm 12.1$ & $63.7 \pm 13.4$ & $<0.001$ \\
\hline$<75$ years & $1508(56.5)$ & $42486(77.4)$ & $<0.001$ & $665(57.4)$ & $18042(76.8)$ & $<0.001$ \\
\hline$\geq 75$ years & $1162(43.5)$ & $12374(22.6)$ & $<0.001$ & $494(42.6)$ & $5455(23.2)$ & $<0.001$ \\
\hline Male & $1687(63.2)$ & $43098(78.6)$ & $<0.001$ & $713(61.5)$ & $18364(78.2)$ & $<0.001$ \\
\hline \multicolumn{7}{|l|}{ Comorbidities } \\
\hline Diabetes mellitus & $1573(58.9)$ & $18516(33.8)$ & $<0.001$ & $676(58.3)$ & 7891(33.6) & $<0.001$ \\
\hline Hypertension & $1757(65.8)$ & $29090(53.0)$ & $<0.001$ & $779(67.2)$ & $12518(53.3)$ & $<0.001$ \\
\hline Coronary artery disease & $682(25.5)$ & $7703(14.0)$ & $<0.001$ & $298(25.7)$ & $3350(14.3)$ & $<0.001$ \\
\hline Prior myocardial infarction & $408(15.3)$ & $6207(11.3)$ & $<0.001$ & 185(16) & 2583(11) & $<0.001$ \\
\hline Heart failure & $563(21.1)$ & $3241(5.9)$ & $<0.001$ & $245(21.1)$ & $1432(6.1)$ & $<0.001$ \\
\hline Chronic kidney disease & $561(21.0)$ & $694(1.3)$ & $<0.001$ & $257(22.2)$ & $298(1.3)$ & $<0.001$ \\
\hline Prior AKI & 296(11.1) & $574(1.1)$ & $<0.001$ & $112(9.7)$ & $246(1.1)$ & $<0.001$ \\
\hline Prior stroke & $585(21.9)$ & $5404(9.9)$ & $<0.001$ & $237(20.5)$ & $2299(9.8)$ & $<0.001$ \\
\hline Peripheral arterial disease & 297(11.1) & $1726(3.2)$ & $<0.001$ & $146(12.6)$ & $804(3.4)$ & $<0.001$ \\
\hline Gout & $256(9.6)$ & $4087(7.5)$ & $<0.001$ & 113(9.8) & $1710(7.3)$ & 0.002 \\
\hline Malignancy & 197(7.4) & $2224(4.1)$ & $<0.001$ & $85(7.3)$ & $920(3.9)$ & $<0.001$ \\
\hline Number of intervened vessels & & & $<0.001$ & & & $<0.001$ \\
\hline 1 & 1918(71.8) & $44974(82.0)$ & & $853(73.6)$ & 19244(81.9) & \\
\hline 2 & $650(24.3)$ & $8846(16.1)$ & & $261(22.5)$ & $3806(16.2)$ & \\
\hline 3 & 102(3.8) & $1040(1.9)$ & & $45(3.9)$ & $447(1.9)$ & \\
\hline Cardiogenic shock & $1787(66.9)$ & $11806(21.5)$ & $<0.001$ & $750(64.7)$ & $4988(21.2)$ & $<0.001$ \\
\hline IABP use & $912(34.2)$ & $5772(10.5)$ & $<0.001$ & $379(32.7)$ & 2354(10) & $<0.001$ \\
\hline Ventilator use & $1257(47.1)$ & $4971(9.1)$ & $<0.001$ & $554(47.8)$ & $2043(8.7)$ & $<0.001$ \\
\hline \multicolumn{7}{|l|}{ Dosage of inotropic medications } \\
\hline Dopamine $\left(\times 10^{3} \mathrm{mg}\right)$ & $3.0 \pm 6.2$ & $0.4 \pm 2.0$ & $<0.001$ & $2.9 \pm 5.8$ & $0.4 \pm 1.8$ & $<0.001$ \\
\hline Norepinephrine (mg) & $5.1 \pm 14.4$ & $0.4 \pm 3.5$ & $<0.001$ & $4.1 \pm 11.1$ & $0.4 \pm 2.9$ & $<0.001$ \\
\hline Epinephrine (mg) & $16.1 \pm 59.2$ & $2 \pm 19.3$ & $<0.001$ & $21.9 \pm 167$ & $1.8 \pm 14.6$ & $<0.001$ \\
\hline \multicolumn{7}{|l|}{ Other medications } \\
\hline Aspirin & $2341(87.7)$ & $51969(94.7)$ & $<0.001$ & $1010(87.1)$ & $22224(94.6)$ & $<0.001$ \\
\hline Clopidogrel & 2452(91.8) & $48920(89.2)$ & $<0.001$ & 1079(93.1) & 21113(89.9) & $<0.001$ \\
\hline B-blocker & $1547(57.9)$ & $34740(63.3)$ & $<0.001$ & $682(58.8)$ & $15039(64)$ & $<0.001$ \\
\hline ACEi/ARB & $1596(59.8)$ & $41719(76.1)$ & $<0.001$ & $672(58)$ & 18023(76.7) & $<0.001$ \\
\hline Calcium channel blocker & $1343(50.3)$ & $16440(30.0)$ & $<0.001$ & $591(51)$ & $6926(29.5)$ & $<0.001$ \\
\hline Statin & $1069(40.0)$ & $26064(47.5)$ & $<0.001$ & $466(40.2)$ & $11342(48.3)$ & $<0.001$ \\
\hline PPI & $828(31.0)$ & $3505(6.4)$ & $<0.001$ & $338(29.2)$ & $1545(6.6)$ & $<0.001$ \\
\hline GP IIb/IIIa & $41(1.54)$ & 1041(1.9) & 0.179 & $18(1.6)$ & $434(1.9)$ & 0.466 \\
\hline Metformin & $224(8.4)$ & $8227(15)$ & $<0.001$ & $95(8.2)$ & $3525(15)$ & $<0.001$ \\
\hline ICU stays (days) & $13.2 \pm 17.5$ & $3.8 \pm 5.3$ & $<0.001$ & $13 \pm 15.8$ & $3.7 \pm 6.3$ & $<0.001$ \\
\hline Hospital stays (days) & $26.7 \pm 34.4$ & $8.5 \pm 14.6$ & $<0.001$ & $28 \pm 41.4$ & $8.4 \pm 11.4$ & $<0.001$ \\
\hline In hospital mortality & $908(34.0)$ & $2,838(5.2)$ & $<0.001$ & $385(33.2)$ & $1,187(5.1)$ & $<0.001$ \\
\hline Major bleeding requiring blood transfusion & 1965(73.6) & 7984(14.6) & $<0.001$ & $863(74.5)$ & $3379(14.4)$ & $<0.001$ \\
\hline
\end{tabular}

ACEi, angiotensin converting enzyme inhibitor; AKI, acute kidney injury; ACS, acute coronary syndrome; ARB, angiotensin II receptor blocker; GP, glycoprotein; IABP, intra-aortic balloon pump; ICU, intensive care unit; PPI, proton pump inhibitor.

\section{Risk model development: full model and reduced model (ADVANCIS)}

As listed in Table 3, the full model contained 16 variables, including age, diabetes mellitus, ventilator use, prior $\mathrm{AKI}$, number of intervened vessels, CKD, IABP use, cardiogenic shock, female sex, prior stroke, peripheral arterial disease, hypertension, coronary artery disease, heart failure, malignancy, and gout. To develop a simple and convenient tool, 8 variables were selected to generate a reduced model, ADVANCIS, on the basis of the regression coefficients[12] (A for age, $\mathrm{D}$ for diabetes mellitus, $\mathrm{V}$ for ventilator use, $\mathrm{A}$ for prior $\underline{\mathrm{A} K I}, \mathrm{~N}$ for number of intervened vessels, $C$ for $C K D$, I for IABP use, and $S$ for cardiogenic shock). VIFs of variables included in the ADVANCIS were less than 2 (ranged from 1.01 to 1.53) which indicated there was no apparent problem of multi-collinearity. 
Table 3. Risk factor analysis for incident $\mathrm{AKI}$ after $\mathrm{PCl}$ for $\mathrm{ACS}$ in training cohort

\begin{tabular}{|c|c|c|c|c|c|c|c|c|}
\hline \multicolumn{5}{|c|}{ Full model } & \multicolumn{4}{|c|}{ Reduced model (ADVANCIS) } \\
\hline Variables & $\beta$ & $\mathrm{aOR}$ & $95 \% \mathrm{CI}$ & $p$ & $\beta$ & $\mathrm{aOR}$ & $95 \% \mathrm{CI}$ & $p$ \\
\hline Age, year & 0.016 & 1.02 & $1.01-1.02$ & $<0.001$ & 0.021 & 1.02 & $1.02-1.03$ & $<0.001$ \\
\hline $\begin{array}{l}\text { Diabetes } \\
\text { mellitus }\end{array}$ & 0.599 & 1.82 & $1.66-2.00$ & $<0.001$ & 0.736 & 2.09 & $1.91-2.28$ & $<0.001$ \\
\hline $\begin{array}{l}\text { Ventilator } \\
\text { use }\end{array}$ & 0.975 & 2.65 & $2.38-2.95$ & $<0.001$ & 1.000 & 2.72 & $2.45-3.02$ & $<0.001$ \\
\hline Prior AKI & 1.317 & 3.73 & $3.09-4.50$ & $<0.001$ & 1.486 & 4.42 & $3.67-5.32$ & $<0.001$ \\
\hline $\begin{array}{l}\text { Number of } \\
\text { intervened } \\
\text { vessels }\end{array}$ & & & & & & & & \\
\hline 2 vs 1 & 0.275 & 1.32 & $1.19-1.46$ & $<0.001$ & 0.298 & 1.35 & $1.21-1.50$ & $<0.001$ \\
\hline 3 vs 1 & 0.651 & 1.92 & $1.51-2.43$ & $<0.001$ & 0.680 & 1.98 & $1.56-2.50$ & $<0.001$ \\
\hline $\begin{array}{l}\text { Chronic } \\
\text { kidney } \\
\text { disease }\end{array}$ & 2.431 & 11.38 & $9.86-13.13$ & $<0.001$ & 2.530 & 12.55 & $10.9-14.46$ & $<0.001$ \\
\hline IABP use & 0.586 & 1.80 & $1.62-2.00$ & $<0.001$ & 0.545 & 1.72 & $1.55-1.92$ & $<0.001$ \\
\hline $\begin{array}{l}\text { Cardiogenic } \\
\text { shock }\end{array}$ & 1.216 & 3.37 & $3.02-3.77$ & $<0.001$ & 1.219 & 3.38 & $3.03-3.78$ & $<0.001$ \\
\hline Female sex & 0.178 & 1.19 & $1.09-1.32$ & $<0.001$ & & & & \\
\hline Prior stroke & 0.114 & 1.12 & $1.00-1.26$ & 0.054 & & & & \\
\hline $\begin{array}{l}\text { Peripheral } \\
\text { arterial } \\
\text { disease }\end{array}$ & 0.392 & 1.48 & $1.27-1.73$ & $<0.001$ & & & & \\
\hline Hypertension & 0.189 & 1.21 & $1.09-1.33$ & $<0.001$ & & & & \\
\hline $\begin{array}{l}\text { Coronary } \\
\text { artery disease }\end{array}$ & 0.011 & 0.99 & $0.88-1.11$ & 0.850 & & & & \\
\hline Heart failure & 0.451 & 1.57 & $1.38-1.79$ & $<0.001$ & & & & \\
\hline Malignancy & 0.043 & 1.04 & $0.87-1.25$ & 0.637 & & & & \\
\hline Gout & 0.024 & 1.02 & $0.87-1.20$ & 0.771 & & & & \\
\hline
\end{tabular}

The AUROC full model and AUROC reduced model for incident AKI were 0.879 (95\% confidence interval [CI]: 0.873-0.886) and $0.874 \quad(95 \% \quad \mathrm{CI}: \quad 0.868-0.881)$, respectively. This result indicated that the reduced model has a discriminatory ability equal to that of the full model (Figure 2).

\section{ADVANCIS score for predicting AKI after PCI for ACS}

The score of each predictor and the risk of incident AKI according to total points are listed in Table 4. A value of 0 was assigned when a factor was absent, and a value of $>0$ was assigned when a factor was present. The scheme of the 8 parameters is described as follows: age score ranged from 0 to 3 , diabetes mellitus was scored 0 or 2 , ventilator use was scored 0 or 2, prior AKI was scored 0 or 3 , number of intervened vessels score ranged from 0 to $2, \mathrm{CKD}$ was scored 0 or 6 , IABP use was scored 0 or 1 , and cardiogenic shock was scored 0 or 3 . The score of each factor was summed to generate the ADVANCIS score, ranging from 0 to 22 points.

A higher ADVANCIS score was associated with a corresponding increase in the risk of AKI $(0.5 \%$ to $98.2 \%)$. A score $\leq 5$ was associated with a probability $<5 \%$, a score $\geq 13$ corresponded to a probability $>50 \%$, and a score $\geq 18$ predicted a probability $>90 \%$.
Table 4. ADVANCIS score and the risk of post-ACS AKI

\begin{tabular}{llll}
\hline Single predictor & & \multicolumn{2}{l}{ Total points and risk (\%) } \\
\hline Risk factor / category & Point & Points total & Risk \\
\hline Age, years & & 0 & 0.5 \\
20 to 39 & 0 & 1 & 0.7 \\
40 to 59 & 1 & 2 & 1.1 \\
60 to 79 & 2 & 3 & 1.6 \\
$\geq 80$ & 3 & 4 & 2.5 \\
Diabetes mellitus & 1 & 5 & 3.7 \\
Ventilator use & 2 & 6 & 5.6 \\
Prior AKI & 3 & 7 & 8.3 \\
Number of intervened vessels & & 8 & 12.2 \\
1 & 0 & 9 & 17.6 \\
2 & 1 & 10 & 24.6 \\
3 & 2 & 11 & 33.4 \\
Chronic kidney disease & 6 & 12 & 43.4 \\
IABP use & 1 & 13 & 54.0 \\
Cardiogenic shock & 3 & 14 & 64.2 \\
& & 15 & 73.3 \\
& & 16 & 80.8 \\
& & 17 & 86.6 \\
& & 18 & 90.8 \\
& & 19 & 93.8 \\
& & 20 & 95.9 \\
& & 21 & 97.2 \\
& & & 98.2 \\
\hline
\end{tabular}

AKI, acute kidney injury; ACS, acute coronary syndrome; IABP, intra-aortic balloon pump

\section{Validation of ADVANCIS model}

The performance of ADVANCIS applied to the validation dataset was satisfactory with an AUROC $_{\text {reduced model }}$ of 0.8624 (95\% CI: 0.8515-0.8733). Moreover, when we restricted the parameter estimates in the validation cohort to be equal to those in the training cohort, the restricted AUROC $_{\text {reduced model }}$ was 0.8621 (95\% CI: $0.8513-0.8730)$, which was comparable with the unrestricted AUROC reduced model ( $P$ of delta of AUROC $=0.4730$ ). This result indicates the generalizability of the ADVANCIS scores (Figure $3)$.

\section{ADVANCIS score for predicting in-hospital mortality}

The ability of the ADVANCIS score to predict the risk of in-hospital mortality was further evaluated. The AUROC of the ADVANCIS score in discriminating in-hospital mortality in the validation dataset was 0.935 (95\% CI: 0.932-0.937) and the optimal cut-off point was 6 , with a sensitivity of $92.4 \%$ and a specificity of $86.3 \%$ (data not shown). We divided the patients into the subgroups low risk, moderate risk, and high risk on the basis of the ADVANCIS score. As illustrated in Figure 4, compared with the low (score 0-5) risk group, the odds ratio of in-hospital mortality in the moderate (score 6-7) and high (score 8-22) risk groups was 45.1 and 121.7, respectively, in the training cohort, and 41.9 and 122.3 , respectively, in the validation cohort. 


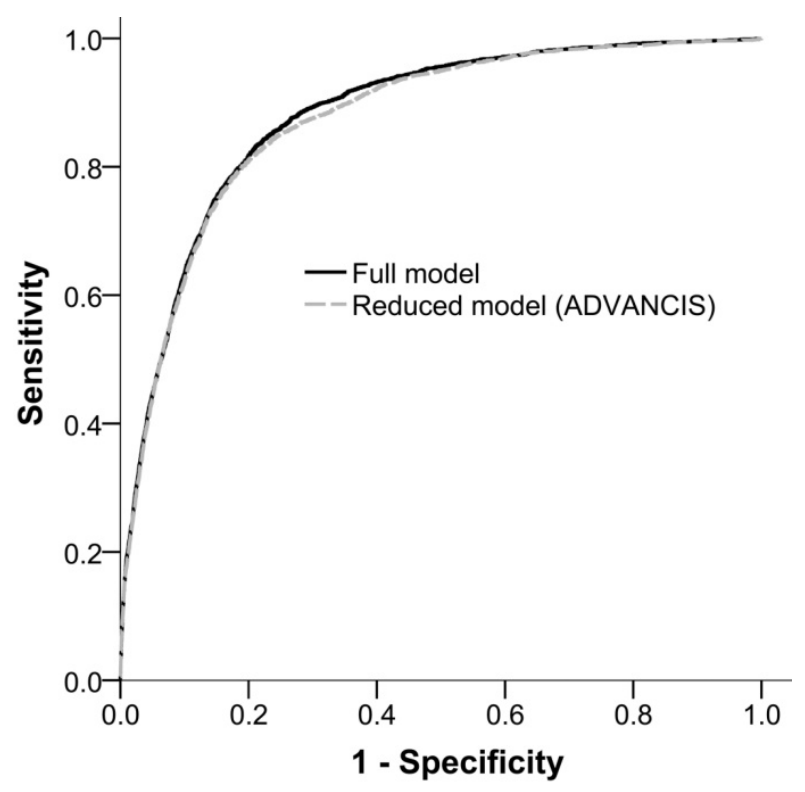

Figure 2. Receiver operation characteristic curves of the full model and the reduced model (ADVANCIS) for incident $\mathrm{AKI}$ after $\mathrm{PCl}$ for $\mathrm{ACS}$ in the training cohort. The AUROCs were $0.879(95 \% \mathrm{Cl}: 0.873-0.886)$ and $0.874(95 \% \mathrm{Cl}$ : $0.868-0.881$ ), respectively.

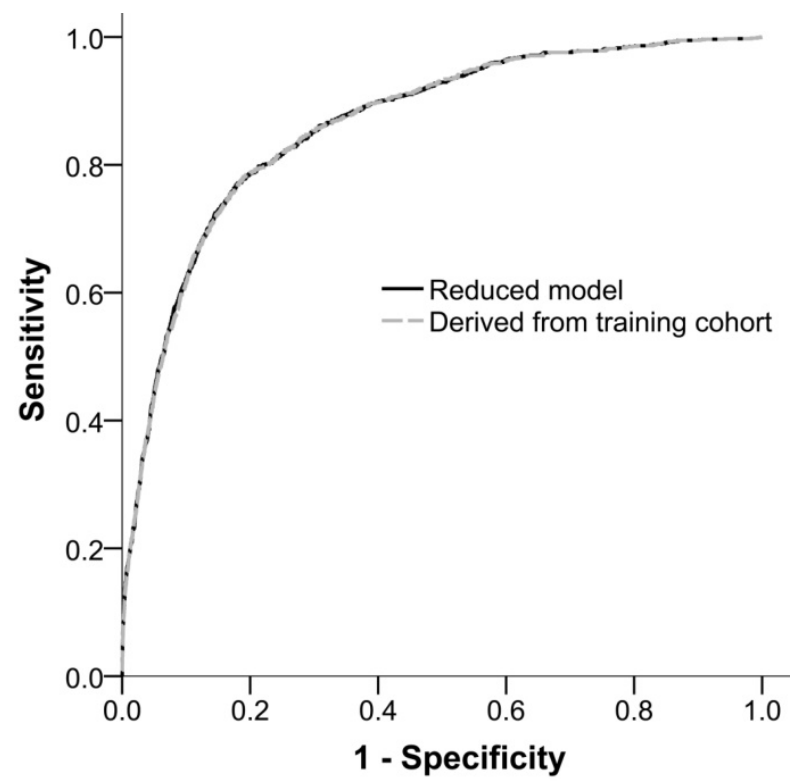

Figure 3. Receiver operation characteristic curves of the reduced model in the validation cohort and the reduced model with parameter estimates derived from the training cohort for incident $\mathrm{AKI}$ after $\mathrm{PCl}$ for $\mathrm{ACS}$. The AUROCs were 0.8624 (95\% Cl: $0.8515-0.8733)$ and 0.8621 (95\% Cl: $0.8513-0.8730)$, respectively.

\section{Discussion}

Previous studies have addressed the incidence and prognostic implications of AKI after ACS [1-3, 6-8]. In our study, the incidence of AKI after receiving PCI for ACS was $4.7 \%$. Consistent with previous studies, the patients with AKI had significantly higher in-hospital morality (33.8\% vs $5.1 \%)$, a longer ICU stay, and a longer hospital stay.
Many studies have reported the risk factors for AKI following ACS [2, 4, 11]. However, the cumulative risk has rarely been discussed, and a widely accepted practical clinical tool to predict the risk of AKI following ACS is lacking. Some studies [13-19] have proposed risk-scoring models to assess the risk of contrast induced nephropathy (CIN) after PCI or coronary angiography. In 2004, Merhan et al proposed a post-PCI CIN risk score based on a cohort of $35.7 \%$ patients with ACS. The score consisted of 8 parameters, namely hypotension, IABP, congestive heart failure, age > 75 years, anaemia, diabetes, contrast media volume, and baseline serum creatinine $>1.5 \mathrm{mg} / \mathrm{dL}$ or estimated glomerular filtration rate < $60 \mathrm{~mL} / \mathrm{min} / 1.73 \mathrm{~m}^{2}$, with satisfactory discriminative power (c statistic 0.67 ) in the validation group [13]. This risk model was recommended by the Kidney Disease: Improving Global Outcome AKI work group [20]. However, CIN after PCI or coronary angiography shares many, but not all, characteristics with post-ACS AKI. ACS itself causes deleterious haemodynamic, immunologic, and neuroendocrine effects on kidney function apart from the effects of contrast medium. Marenzi et al also proposed a model for CIN prediction in patients with acute myocardial infarction receiving primary PCI. The model consisted of 5 parameters, namely age $>75$ years, anterior wall myocardial infarction, time to reperfusion $>6$ hours, contrast agent volume $>300$ $\mathrm{mL}$, and IABP use [15]. Marenzi's group further proposed a risk model to predict AKI after ACS by using 4 variables, namely age, left ventricular ejection fraction, serum concentration, and ST-segmental myocardial infarction [21].

To our knowledge, our study is the first to develop and validate a risk prediction model specific for AKI following ACS and PCI, based on a large nationwide cohort. The risk factor analysis revealed 16 variables as risk factors, including age, diabetes mellitus, ventilator use, prior AKI, number of intervened vessels, CKD, IABP use, cardiogenic shock, female sex, peripheral arterial disease, hypertension, and heart failure. We propose the ADVANCIS score, namely 5 patient-related characteristics, age, diabetes mellitus, prior AKI, CKD, and cardiogenic shock, and 3 procedure-related characteristics, number of intervened vessels, ventilator use, and IABP use. The discriminative power of the ADVANCIS model is equal to that of the full model for predicting AKI following ACS and PCI.

Among the 8 variables, age, diabetes mellitus, prior AKI, CKD, and shock are all known universal risk factors for AKI. The number of intervened vessels not only reflects the severity of coronary artery disease but also provides an estimate of the amount of 
administrated contrast media. IABP use not only is a marker of significant haemodynamic instability but also potentially confers additional hazards to the kidneys by causing atheroemboli during the procedure or occluding renal blood flow, if the pump is malpositioned [13].

The ADVANCIS score provides an incremental risk stratification, and enables clinicians to quickly and accurately predict the risk of AKI after PCI for ACS. A score of $\leq 5$ was associated with a probability of $<5 \%$, a score of $\geq 13$ corresponded to a probability of $>50 \%$, and a score of $\geq 18$ predicted a probability of $>90 \%$. High-risk patients require frequent monitoring; prophylactic strategies, including avoidance of nephrotoxic agents; early intervention to reduce the AKI severity; or prompt management including timely renal-replacement therapy.

AKI is a crucial risk factor for short-term and long-term mortality [22]. Our study showed that the ADVANCIS model had excellent discriminative power in predicting in-hospital mortality. An ADVANCIS score $\geq 6$ is associated with significantly higher in-hospital mortality in patients with ACS after they receive PCI. ADVANCIS scores of $\leq 5$ (low risk), 6-7 (moderate risk), and $\geq 8$ (high risk) were associated with probabilities of in-hospital mortality of $<1 \%,<20 \%$, and $<40 \%$, respectively.

To further evaluate the potential of the ADVANCIS model in facilitating clinical decisionmaking and improving patient outcomes, further prospective validation is necessary. The incorporation of novel biomarkers or other clinical parameters may provide additional prognostic value and warrants further investigation.

Despite the large sample size, a central limitation of this study was its retrospective nature. Information about the type of ACS, such as STEMI or NSTEMI, was not available. Laboratory data of blood or urine tests, fluid status, urine output, and left ventricular ejection fraction could not be assessed. Information regarding the type and volume of contrast medium, and the prophylactic strategy was lacking. Finally, our study did not evaluate long term mortality and renal outcome.

In conclusion, the ADVANCIS score is a potentially useful clinical tool to assess the risk of incident AKI and hospital mortality after PCI for ACS, thereby enabling prompt prevention and intervention.

\section{Methods}

\section{Data collection}

Retrospective data were collected from the Taiwan National Health Insurance Research Database (NHIRD), which was established by the Taiwan National Health Insurance Administration and covers medical benefit claims for more than $99 \%$ of the more than 23 million residents of Taiwan [23]. The NHIRD provides comprehensive and accurate records of beneficiaries, including ambulatory visits, inpatient care, disease diagnosis codes, and medication prescriptions. All clinical diagnoses are recorded according to the International Classification of Diseases, Ninth Revision, Clinical Modification (ICD-9-CM) codes. The records and information of the patients were deidentified to ensure patient anonymity. The NHIRD has been widely used in epidemiology studies of cardiovascular disease and cerebral vascular disease [24-26]. The agreement of comorbidity coding of NHIRD with traditional electronic medical records has been validated [27]. The Institutional Review Board at Linkou Chang Gung Memorial Hospital approved this study and waived the requirement for informed consent.

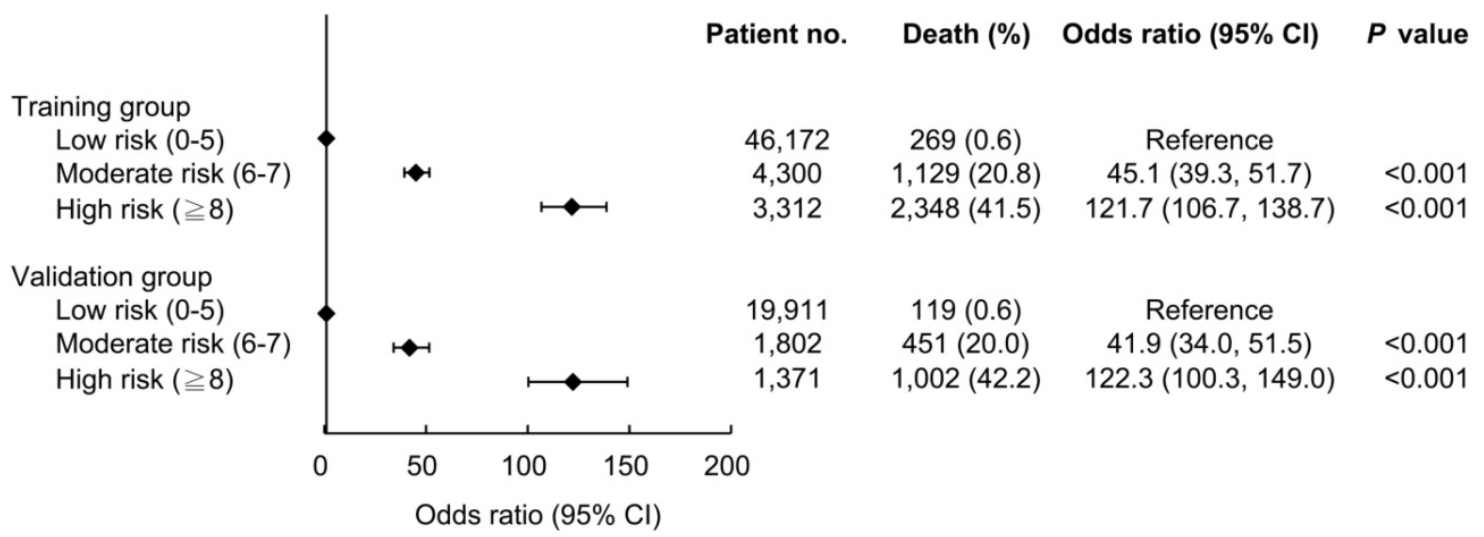

Figure 4. The associations between ADVANCIS score subgroups and risk of in-hospital mortality. 


\section{Study populations}

As illustrated in Figure 1, this study identified 201,526 patients who were admitted with a diagnosis of ACS (ICD-9-CM 410) between January 1, 1997 and December 31, 2011. This coding was validated in a previous study and achieved a positive predicted value of $93 \%$ [24]. Among them, 85,008 patients receiving PCI were enrolled. We excluded patients with (1) an unknown sex ( $\mathrm{n}=46),(2)$ age $<18$ years ( $\mathrm{n}$ $=6$ ), and (3) end-stage renal disease requiring long-term dialysis (ICD-9-CM 585 and corresponding catastrophic illness certificate) $(n=2,770)$. Finally, 82186 eligible patients were enrolled and randomly divided according to a 7:3 ratio to generate a training and validation cohort, respectively.

\section{Outcomes and comorbidities}

The index hospitalization was defined as the date on which patients were admitted for ACS. The primary outcome was incident AKI (ICD-9-CM 584.9) with or without de novo dialysis during index admission. The disease code of AKI was validated in a previous study and achieved a sensitivity of $92 \%$ and a positive predicted value of $100 \%$ [27]. De novo dialysis was defined as dialysis initiated during the index admission. The secondary outcome was mortality during the index admission and the definition was also reported [27]. Cardiogenic shock was identified as (1) the use of $>400 \mathrm{mg}$ of dopamine or (2) the use of $>0 \mathrm{mg}$ of epinephrine or norepinephrine during the index admission $[28,29]$.

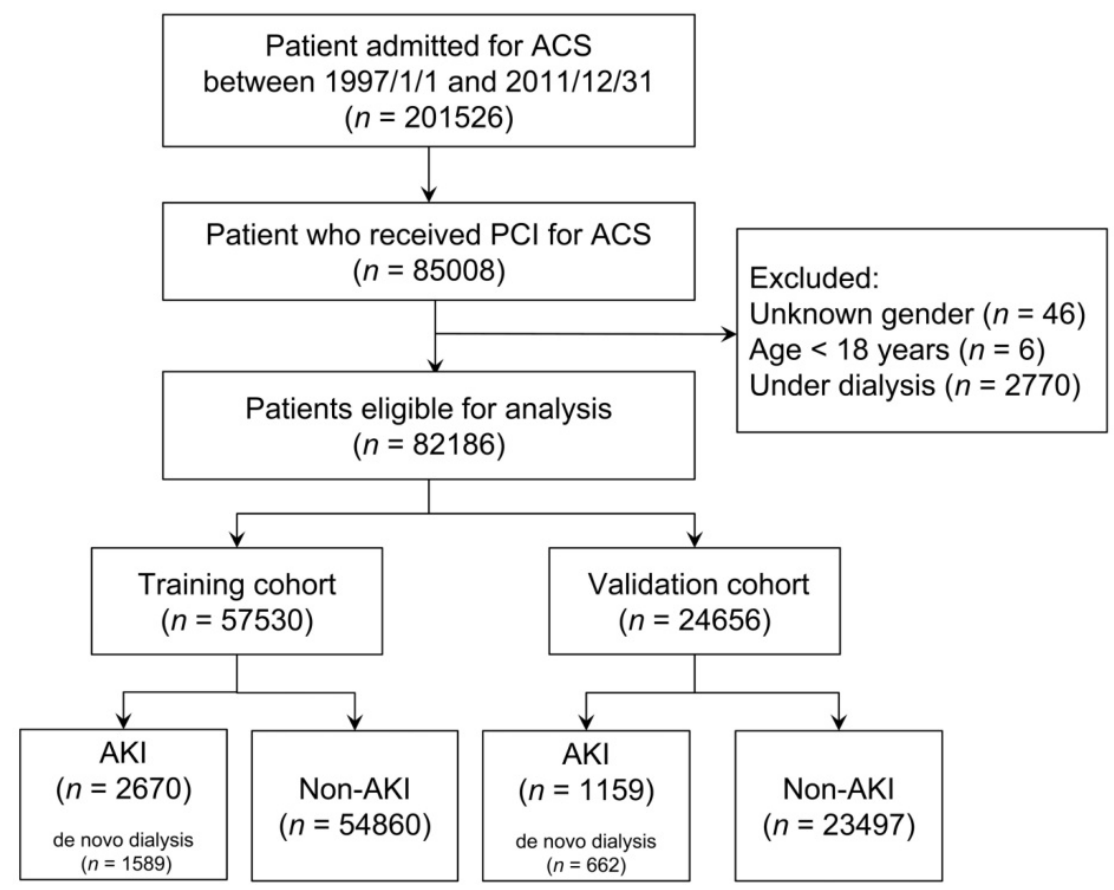

Figure 1. Flow diagram of selection of study population

\section{Statistical analysis}

The distribution of baseline characteristics and clinical information was compared between the patients with and those without AKI by using the Student $t$ test for continuous variables and the chi-square test for categorical variables in the training and validation cohorts. In the next step, we developed a multivariable logistic model (named full model) including baseline characteristics (i.e., sex, age, comorbidities, and major procedures during index admission) as explanatory variables. Subsequently, we selected clinically relevant variables to develop a parsimonious model (named reduced model or ADVANCIS) based on the training cohort data. The multi-collinearity among predictors in reduced model was tested using variance inflation factor (VIF). To assess the extent of loss in discriminating incident AKI attributable to the unselected variables (i.e., sex or prior stroke), we compared the areas under the receiver operation characteristic curve (AUROC) between the full and reduced models [30].

According to the results of the multivariable logistic model based on the training cohort data, we calculated a simplified point system [12] to demonstrate the associations between explanatory variables (covariates) and incident AKI. The points system rounds off the regression coefficients derived from the multivariable logistic model. Firstly, we identified a continuous predictor (i.e. age) with a wide range of values as the reference variable, categorized it into relevant categories. Furthermore, we obtained reference values for each category and categorized the other predictors. Finally, the reference value of each predictor category was calculated according to the value of its regression coefficient relative to that of the reference variable.

Furthermore, to evaluate the generalizability of the reduced model (ADVANCIS), we compared the AUROCs between the validation cohort (estimates were not restricted) and a validation cohort that was derived from the training cohort (estimates of validation cohort were restricted to being equal to those of the training cohort). A $P$ value of $<0.05$ was considered statistically significant. Data analysis as well as random sampling were conducted using IBM SPSS 22 (IBM SPSS, Armonk, NY, USA: IBM Corp). 


\section{Acknowledgements}

The authors thank Alfred Hsing-Fen Lin and all the participating patients of the Kidney Research Center of Chang Gung Memorial Hospital, Linkou, Taiwan.

\section{Sources of Funding}

This research was supported by a research grant from Chang Gung Medical Foundation (BMRPD97, CORPG5G0071, CIRPG3B0043). Dr. C-H Chang was supported by the Ministry of Science and Technology (MOST 103-2314-B-182A-018-MY3).

\section{Author Contributions}

Pei-Chun Fan and Tien-Hsing Chen contributed in drafting the manuscript. Cheng-Chia Lee, Tsung-Yu Tsai and Yung-Chang Chen collected and analyzed the data. Chih-Hsiang Chang conceived and designed the study. All authors read and approved the final manuscript.

\section{Competing Interests}

The authors have declared that no competing interest exists.

\section{References}

1. Bruetto RG, Rodrigues FB, Torres US, Otaviano AP, Zanetta DM, Burdmann EA. Renal function at hospital admission and mortality due to acute kidney injury after myocardial infarction. PLoS One. 2012; 7: e35496.

2. Fox CS, Muntner P, Chen AY, Alexander KP, Roe MT, Wiviott SD. Short-term outcomes of acute myocardial infarction in patients with acute kidney injury: a report from the national cardiovascular data registry. Circulation. 2012; 125: 497-504

3. Parikh CR, Coca SG, Wang Y, Masoudi FA, Krumholz HM. Long-term prognosis of acute kidney injury after acute myocardial infarction. Arch Intern Med. 2008; 168: 987-95.

4. Rodrigues FB, Bruetto RG, Torres US, Otaviano AP, Zanetta DM, Burdmann EA. Incidence and mortality of acute kidney injury after myocardial infarction: a comparison between KDIGO and RIFLE criteria. PLoS One. 2013; 8: e69998.

5. Jose P, Skali H, Anavekar N, Tomson C, Krumholz HM, Rouleau JL, et al. Increase in creatinine and cardiovascular risk in patients with systolic dysfunction after myocardial infarction. J Am Soc Nephrol. 2006; 17: 2886-91.

6. AlFaleh HF, Alsuwaida AO, Ullah A, Hersi A, AlHabib KF, AlNemer K, et al. The prognostic impact of in-hospital worsening of renal function in patients with acute coronary syndrome. International journal of cardiology. 2013; 167: 866-70.

7. Chawla LS, Kimmel PL. Acute kidney injury and chronic kidney disease: an integrated clinical syndrome. Kidney Int. 2012; 82: 516-24.

8. Coca SG, Singanamala S, Parikh CR. Chronic kidney disease after acute kidney injury: a systematic review and meta-analysis. Kidney Int. 2012; 81: 442-8.

9. Ronco C, Haapio M, House AA, Anavekar N, Bellomo R. Cardiorenal syndrome. Journal of the American College of Cardiology. 2008; 52: 1527-39.

10. Marenzi G, Cosentino N, Bartorelli AL. Acute kidney injury in patients with acute coronary syndromes. Heart. 2015; 101: 1778-85.

11. Amin AP, Spertus JA, Reid KJ, Lan X, Buchanan DM, Decker C, et al. The prognostic importance of worsening renal function during an acute myocardial infarction on long-term mortality. Am Heart J. 2010; 160: 1065-71.

12. Sullivan LM, Massaro JM, D'Agostino RB, Sr. Presentation of multivariate data for clinical use: The Framingham Study risk score functions. Stat Med. 2004; 23: 1631-60.

13. Mehran R, Aymong ED, Nikolsky E, Lasic Z, Iakovou I, Fahy M, et al. A simple risk score for prediction of contrast-induced nephropathy after percutaneous coronary intervention: development and initial validation. Journal of the American College of Cardiology. 2004; 44: 1393-9.

14. Silver SA, Shah PM, Chertow GM, Harel S, Wald R, Harel Z. Risk prediction models for contrast induced nephropathy: systematic review. BMJ. 2015; 351: h4395.

15. Marenzi G, Lauri G, Assanelli E, Campodonico J, De Metrio M, Marana I, et al. Contrast-induced nephropathy in patients undergoing primary angioplasty for acute myocardial infarction. Journal of the American College of Cardiology. 2004; 44: 1780-5.

16. Gurm HS, Seth M, Kooiman J, Share D. A novel tool for reliable and accurate prediction of renal complications in patients undergoing percutaneous coronary intervention. Journal of the American College of Cardiology. 2013; 61: 2242-8.

17. Chen YL, Fu NK, Xu J, Yang SC, Li S, Liu YY, et al. A simple preprocedural score for risk of contrast-induced acute kidney injury after percutaneous coronary intervention. Catheter Cardiovasc Interv. 2014; 83: E8-16.

18. Maioli M, Toso A, Gallopin M, Leoncini M, Tedeschi D, Micheletti C, et al. Preprocedural score for risk of contrast-induced nephropathy in elective coronary angiography and intervention. J Cardiovasc Med (Hagerstown). 2010; 11: 444-9.

19. Liu Y, Liu YH, Chen JY, Tan N, Zhou YL, Li HL, et al. A simple pre-procedural risk score for contrast-induced nephropathy among patients with chronic total occlusion undergoing percutaneous coronary intervention. International journal of cardiology. 2015; 180: 69-71.

20. Lameire N, Kellum JA, Group KAGW. Contrast-induced acute kidney injury and renal support for acute kidney injury: a KDIGO summary (Part 2). Crit Care. 2013; 17: 205.

21. Marenzi G, Cabiati A, Bertoli SV, Assanelli E, Marana I, De Metrio M, et al. Incidence and relevance of acute kidney injury in patients hospitalized with acute coronary syndromes. Am J Cardiol. 2013; 111: 816-22.

22. Bellomo R, Kellum JA, Ronco C. Acute kidney injury. Lancet. 2012; 380: 756-66.

23. Hsing AW, Ioannidis JP. Nationwide Population Science: Lessons From the Taiwan National Health Insurance Research Database. JAMA Intern Med. 2015; 175: 1527-9.

24. Cheng $\mathrm{CL}$, Lee $\mathrm{CH}$, Chen PS, Li YH, Lin SJ, Yang YH. Validation of acute myocardial infarction cases in the national health insurance research database in taiwan. J Epidemiol. 2014; 24: 500-7.

25. Cheng CL, Kao YH, Lin SJ, Lee CH, Lai ML. Validation of the National Health Insurance Research Database with ischemic stroke cases in Taiwan. Pharmacoepidemiol Drug Saf. 2011; 20: 236-42.

26. Lee CH, Cheng CL, Lin LJ, Tsai LM, Yang YH. Epidemiology and predictors of short-term mortality in symptomatic venous thromboembolism. Circ J. 2011; 75: 1998-2004.

27. Cheng $\mathrm{CL}$, Chien $\mathrm{HC}$, Lee $\mathrm{CH}$, Lin SJ, Yang $\mathrm{YH}$. Validity of in-hospital mortality data among patients with acute myocardial infarction or stroke in National Health Insurance Research Database in Taiwan. International journal of cardiology. 2015; 201: 96-101.

28. Mao CT, Wang JL, Chen DY, Tsai ML, Lin YS, Cherng WJ, et al. Benefits of Intraaortic Balloon Support for Myocardial Infarction Patients in Severe Cardiogenic Shock Undergoing Coronary Revascularization. PLoS One. 2016; 11: $\mathrm{e} 0160070$.

29. Chen DY, Mao CT, Tsai ML, Chen SW, Lin YS, Hsieh IC, et al. Clinical outcomes of drug-eluting stents versus bare-metal stents in patients with cardiogenic shock complicating acute myocardial infarction. International journal of cardiology. 2016; 215: 98-104.

30. DeLong ER, DeLong DM, Clarke-Pearson DL. Comparing the areas under two or more correlated receiver operating characteristic curves: a nonparametric approach. Biometrics. 1988; 44: 837-45. 